\title{
The foundations of collaborative group decisions
}

\author{
Ralph L. Keeney \\ The Fuqua School of Business, \\ Duke University, \\ 1 Towerview Drive, Durham, NC 27708, USA \\ E-mail: keeney@duke.edu
}

\begin{abstract}
Collaborative engineering requires collaborative group decisions. This paper presents foundations for a collaborative group decision model, and offers suggestions on how to implement such a model. Specifically, decision analysis techniques are explained to provide a sound foundation and a helpful tool to guide collaborative group decisions. These techniques do not oversimplify the realities of collaborative group decisions. They explicitly knowledge differences of judgements and values among group members and offer a constructive framework to account for these differences in the decision process.
\end{abstract}

Keywords: group decision; collaboration; decision analysis.

Reference to this paper should be made as follows: Keeney, R.L. (2009)

'The foundations of collaborative group decisions', Int. J. Collaborative Engineering, Vol. 1, Nos. 1/2, pp.4-18.

Biographical notes: R.L. Keeney is a member of the National Academy of Engineering in USA. His areas of expertise are decision analysis, risk analysis, and management decision-making. He is an authority on decision making with multiple objectives. He has contributed substantially toward the development of decision analysis and risk analysis. His experience includes corporate management problems, risk analyses, energy policy, large-scale siting studies (e.g., airports, power plants), and environmental studies. He has been a consultant for several organisations including Fair Isaac, Seagate Technology, American Express, British Columbia Hydro, Pacific Gas and Electric, and the Electric Power Research Institute.

\section{Introduction}

By making decisions you purposely influence your own life and the world around you. If you make no decisions, you have no direct control on anything and life just happens to you. So clearly decisions are important and they are important to make well.

A standard way to categorise decisions is as individual decisions or group decisions. The key distinction is whether only one person or more than one has complete control over the choices of alternatives. With group decisions, more than one person has control to influence the choice of alternatives.

The focus in this paper is on collaborative group decisions. Not all group decisions are collaborative group decisions. Specifically, I define collaborative group decisions to exclude three classes of group decisions: negotiations, risk sharing arrangements, 
and voting systems. For some group decisions, all of those who influence the choice do not end up sharing the same consequences of that choice. Obvious examples are many negotiations and business arrangements designed to share risks. If a buyer and seller negotiate successfully on a house purchase, the seller gets money and the buyer gets a house. The sets of alternatives that the buyer and seller have to choose from are different in this case; the seller can sell or not and the buyer can buy or not given the details of the proposed arrangement. With sharing risks of investments, three individuals may jointly choose among several investment alternatives, but the consequences to each may not be the same depending on their risk sharing arrangement.

Another class of group decisions that I do not include as collaborative group decisions are standard voting procedures. Here there is typically no interaction among the decision makers (i.e., the voters) or their choices (i.e., votes). They each decide how to vote and the winner is selected by tabulating the votes according to the procedure being used (e.g., the alternative with the maximum number of votes wins). With elections, decision makers select among the same alternatives and experience the same consequences, namely the elected winner.

Collaborative group decisions as defined in this paper require three properties:

- the responsibility for making decisions rests with a group of two or more members

- all of whom experience the same consequences of the decision

- who jointly must interact to select alternatives.

Note that the third property does not require that only one alternative is chosen or that all of the decision makers control the selection of each of the alternatives. An engineering design decision may involve the choice of many alternatives that influence function, costs, safety, and form. Different members of the design team may have responsibility individually or jointly for the different required decisions. Such a decision is a collaborative group decision as the three properties above are satisfied.

Collaborative group decisions are complex if for no other reason than more than one individual has influence on the decisions. But there are often other factors that add additional complexity. These factors include multiple objectives to be achieved, significant uncertainties about consequences, and numerous alternatives. Furthermore, the individuals included in the decisions may not agree on the alternatives or objectives, on the prioritising of the objectives even if they agree on the set, or on how well the alternatives meet the various objectives. Thus an analytical model to facilitate communication and inform the decision makers can be useful.

This paper presents foundations for a collaborative group decision model and offers suggestions on how to implement such a model. It is organised as follows. Section 2 presents the reasons for why an analysis of a collaborative decision problem may be useful. Section 3 presents the underlying assumptions of decision analysis for collaborative group decisions and argues that it is an appropriate approach for such choices Section 4 provides guidelines and procedures to implement a decision analysis model on collaborative group decisions, where group members may disagree on possible consequences of decisions and/or values. The important topic of aggregating preferences of individuals is summarised in Section 5. Section 6 discusses uses of the model. 


\section{Basic tenets for analysing collaborative group decisions}

The reasonableness of any analytical approach to analyse decisions depends on the appropriateness of its assumptions, the principles it incorporates, and the quality of information that can be gathered that is required for the analysis. The usefulness of the analysis is indicated by the quality of the insights that it can provide. This section and Section 3 discuss the assumptions and principles. Section 4 discusses procedures to obtaining quality information.

Decision analysis provides a solid foundation to analysing collaborative group decisions. Intuitively, I think of decision analysis as a formalisation of common sense for decision problems that are too complex for the informal use of common sense. More specifically, decision analysis can be defined as a set of logical axioms and a methodology and collection of systematic procedures, based upon those axioms, for responsibly analysing the complexities inherent in decision problems.

In specifying any set of assumptions, there is always the question of where to begin and what to include. Typically, sets of assumptions for decision methodologies specify only what is to be done and how it can theoretically be done. We shall also include guidelines about why it should be done and how to do it in practice. In doing this we hope to improve the ability of a collaborative group to appraise the appropriateness of decision analysis for its decisions.

There are four basic tenets that a collaborative group must hold in order for decision analysis to be of use. There tenets are essentially the assumptions for why do an analysis. These basic tenets are the following:

- Basic Tenet 1. The collaborative group has an important decision to make.

- Basic Tenet 2. The decision is complex, meaning it involves high stakes, complicated structure, and/or no overall experts.

- Basic Tenet 3. The analysis should address the complexity inherent in the decision.

- Basic Tenet 4. The analysis can facilitate both communication and the appraisal of the various alternatives.

It is difficult to find fault with these tenets if a collaborative group faces a difficult choice. This directly satisfies Tenets 1 and 2 by definition. To elaborate on the complexity of the decision, high stakes means that there is potential for both significant upside and downside to the consequences and complicated structure means there are many factors to consider in the decision and that the relationships between and among factors are intertwined and/or involve uncertainties. Because of this, there are no experts on all aspects relevant to the decision who, in theory, may be able to logically process all of the information without analyses.

Basic Tenet 4 is easy to accept if one assumes that the analysis is of high quality. This relates to basic Tenet 3 since being of high quality requires that the complex aspects of the decision be addressed in the analysis. The qualifier can in Tenet 4 is because there is no guarantee that an analyses will be of high quality. Of course, the analysts and the collaborative group, who may be the same people, can greatly influence the quality of the analysis. 


\section{The foundations of decision analysis}

The foundations of decision analysis provide a logical basis for structuring and analysing any decision problem. The axioms that specify the foundations are stated in slightly different manners in von Neumann and Morgenstern (1947), Savage (1954) and Pratt et al. (1964). Rather than repeat a formal statement of these axioms, I will state a less formal and more operational set of assumptions that use the same concepts with an extension to include structuring the decision problem.

The first two assumptions concern structuring the decision problem.

Assumption 1 (Specification of Objectives). A list of the objectives to be achieved by the decision of concern can be generated. The reason for being interested in any decision is its objectives. If the set of objectives has not been generated, then there is no basis to even think about which alternatives may be better than which others and why this is the case. It follows that if the objectives are not specified, members of the collaborative group should put forth effort to generate the appropriate objectives.

Assumption 2 (Identification of Alternatives). A set of alternatives, which means two or more, can be identified for the decision. It is by the choice of the alternative that a decision is made. Without at least two alternatives, there can be no decision. There are natural relationships between the objectives and alternatives, as the objectives provide a basis for both creating and evaluating alternatives, and the potential consequences of the alternatives indicate the breadth of objectives that should be included.

Once sets of objectives and alternatives are articulated, it is important to describe how well each alternative meets the various objectives. This is done by describing the possible consequences of each alternative.

Assumption 3 (Description of Consequences). Possible consequences of each alternative can be specified, which indicate the complete list of consequences that may occur for each alternative and their probabilities of occurrence.

As an aspect of evaluating alternatives, it is necessary to know how good each of the possible consequences is. This gives rise to an assumption about the value of each possible consequence.

Assumption 4 (Qualification of Values). The relative desirability of each possible consequence can be specified. Technically, it is necessary to specify the relative desirabilities with a utility function (von Neumann and Morgenstern, 1947) which allows one to evaluate the expected utility of each alternative. It is the expected utility that should be used to evaluate alternatives consistently with the decision analysis principles of logic stated in the next three assumptions.

Assumption 5 (Comparison of Alternatives). If two alternatives could each result only in the same two possible consequences, the alternative yielding the higher chance of the preferred consequence should be preferred.

Assumption 6 (Transitivity of Preferences). If a first alternative is preferred to a second alternative and the second alternative is preferred to a third alternative, then the first alternative should be preferred to the third alternative. 
Assumption 7 (Substitution of Consequences). If an alternative is modified by replacing some of its consequences with another set of consequences indifferent to those being replaced, then the original and modified alternatives should be equally preferred.

Assumption 5 is necessary to indicate how various alternatives should be compared. Assumptions 6 and 7 are the consistency assumptions that are used to reduce complex alternatives, meaning those with more than two possible consequences, to an equivalently preferred simple alternative of the form referred to in Assumption 5. Once it is in this form, then it can easily be compared with other alternatives.

The fundamental implication of these assumptions is the following: If the assumptions are accepted, then the expected utility of each alternative indicates its desirability and alternatives with higher expected utilities should be preferred. Use of this result is discussed in Section 6. Specifically the ranking of alternatives provided by the expected utilities is not the basic result of an analysis. Rather, the importance of an analysis is the insights it provides, and for this a thorough sensitivity analysis that varies all of the inputs to the analysis is worthwhile.

\section{Appraisal of the foundations}

The assumptions of decision analysis concern all the information that is needed to logically structure and analyse a decision. They do not address how to get this information. The assumptions are very general and conform to common sense. This has two important implications.

First, most people want important decisions with which they are concerned to be made consistent with these assumptions. Naturally, it is reasonable to begin with a clear understanding of objectives and alternatives. Then, to appraise alternatives, it is necessary to know how well the alternatives might meet each of the objectives. It is also necessary to know the relative desirability of the different degrees to which these objectives are met. The consistency assumptions to most people 'just make sense' as a guide to how decisions should be made.

Second, because decisions are not over-specified or over-simplified by these assumptions, gathering the information necessary for quality analysis is difficult. This is especially the case for collaborative decisions. This topic is addressed in Section 4. Many methodologies intended to facilitate or inform decision making begin by assuming that the sets of objectives, or even an objective function, are pre-specified. The decision analysis approach includes these crucial phases of decision making as part of the process.

Finally, and very importantly, the assumptions of decision analysis stated above do not require or refer to a decision maker. The assumptions address how the complexity of the decision problem should be addressed, not how the process to address the complexity should occur. The decision maker or decision makers are part of the process. It is perhaps more difficult to get the information needed to implement the assumptions for a particular decision when that decision is faced by a collaborative group rather than an individual. On the positive side, there is the potential for greater insights. Both of these considerations are addressed in the next two sections. 


\section{Implementing the principles of decision analysis for collaborative group decisions}

It is useful to summarise the information required to analyse a decision problem using the decision analysis principles. This will allow us to succinctly discuss how the collaborative group should create and gather this information. This requires some notation.

To structure a decision, we need a set of alternatives $A_{j}, j=1, \ldots, J$ and a set of objectives $O_{i}, i=1, \ldots, N$ to be achieved by these alternatives. To describe consequences, we need to identify an attribute $X_{i}, i=1, \ldots, N$, to measure achievement on the corresponding objective $O_{i}$. Then we can describe a possible consequence by $x=\left(x_{1}, \ldots, x_{N}\right)$ of an alternative, where $x_{i}$ is a specific level of $X_{i}$. Examples of an objective are 'maximise profits' and 'insure production quality', which can be measured by attributes 'annual profit in dollars' and "failure rate for a quality test." Corresponding levels of the attributes might be '\$29 million' and a '0.013 failure rate'.

For each alternative $A_{j}$, one needs to determine a probability distribution function $p_{j}(x)$ over the possible consequences that specifies the probability of consequence $x$ occurring if $A_{j}$ is selected. To quantify values to evaluate these consequences, a utility function $u(x)$ must be determined.

Given all of the above information, it is straightforward to evaluate the relative desirability of alternatives by calculating the expected utility $U_{j}$ of each using

$$
U_{j}=\int p_{j}(x) u(x) \mathrm{d} x, \quad j=1, \ldots, J .
$$

Various sources talk about how to implement decision analysis for a single decision maker. An excellent reference is Clemen and Reilly (2001). When a group has responsibility for a decision, even though the information required is the same, the task is more involved mainly because members of the group may not agree on the information. This section discusses how to collect the information needed to use decision analysis for collaborative decisions. It also suggests procedures to better understand any differences of judgements and values held by the various group members, which we think would often be the case, and resolve or reduce these differences. Prior to discussing separately the information needed about objectives, alternatives, consequences, and values, we will consider validating the assumptions.

\section{Validating assumptions}

The assumptions of decision analysis provide the foundation of the approach in terms of basic principles. If the members of the collaborative group agree with the assumptions, it follows that the approach should be followed in order to make decisions consistent with the assumptions.

There are two general situations to consider. In one, the collaborative group has responsibility for making a decision within an organisation. Examples are a design team for a new product in a company, a company wide task force with responsibility for selecting a location for a major facility (e.g., power plant, regional distribution centre), or a branch of government agency charged with specifying a new policy. A guideline from the management of the organisation for the collaborative group may be to do an analysis consistent with the assumptions of decision analysis to inform the 
decision making process. In this situation, the members of the collaborative group should first understand the assumptions and proceed.

The other situation concerns an autonomous collaborative group that has no management guidelines and can set their own rules for the decision being faced. Here, the group should discuss the assumptions to see if they are reasonable for evaluating alternatives. Based on my experience, there are few objections to the assumptions as they basically state what many consider to be common sense. If you want to make a good decision, who would not want to have a clear understanding of the objectives and alternatives? Why would not you want to know how well the alternatives achieve the objectives? And you really have no choice in whether to address the value issues, so why not do it consistently and explicitly because the relative values of the consequences is the reason you even care about the decision. The consistency assumptions are reasonable for prescriptive choice. Descriptively, individuals often express preferences that contain intransitivities (Tversky, 1969), but most people state they do not want intransitivities in their evaluation of important decisions.

When individuals say they have objections to one or more or the basic assumptions of decision analysis, it is usually not really an objection to the assumptions, but rather to how they may be used or misused. For example, an individual in the collaborative group may not want to include certain alternatives because they are obviously inferior to other alternatives. However, if this is the case, a quality analysis should bear it out. Other individuals in the collaborative group, who wanted to include that alternative, may in this case learn that their intuition about its desirability was over-estimated. Issues such as these are considered in detail later in this section.

An individual may think that the assumptions are unreasonable for certain decisions. One such decision may concern a group with the responsibility to select a national policy to decide who gets the limited inoculations against a deadly viral disease. The individual may believe that this is a moral issue and that moral issues are not subject to analysis. Even if this view is accepted, the issue in accepting the assumptions or not is whether they are appropriate for the specific decision being faced, not whether it is appropriate for all decisions.

\section{Specifying objectives}

Each member of the collaborative group can write down the set of objectives he or she feels are appropriate for the decision. Suggestions for stimulating the thought processes to do this well are found in Keeney (1992). The collection of all of these objectives should be the set used in the collaborative decision. This naturally insures all the objectives relevant to each member of the group are included. Note that this does not require that members agree on which objectives are important or even relevant. These issues, importance and relevance, are dealt with in expressing relative values for the objectives.

Once a complete set of objectives for the decision is produced, it should be organised. The first aspect of this is to combine what are essentially the same objectives. For example, if different members of a collaborative group wrote 'make money', 'meet our profit goal', 'earn a fair profit', 'profit', and 'maximise profits', these could all be combined under the objective 'maximise profits'.

Finally, one wants to separate the objectives into those that are fundamental objectives to the decision, meaning those stating the reason for interest in the decision, and those that are means objectives, meaning those that influence how well the 
fundamental objectives are achieved. For example, in evaluating alternatives to improve air quality, one objective would be to 'minimise emissions'. This is a means objective to "minimise pollutant concentrations", which is a means influencing "minimise doses to humans". This is naturally a means to 'maximise health effects', which would likely be a fundamental objective of the decision. Another fundamental objective of the decision would be to 'minimise the costs' of improving the air quality. These two fundamental objectives naturally conflict, meaning you cannot simultaneously maximise each. If they did not conflict to some degree, they would essentially be the same objective.

Balancing the degree to which each objective contributes to the overall desirability of the alternatives is considered when quantifying values. The values need only to be quantified over fundamental objectives. The means-ends relationships usually have a factual basis, as pollutant emissions do influence pollutant concentrations, and members of the group should not have many disagreements about these relationships. They may disagree about the strength of the influence, which is addressed in describing consequences of alternatives. There may be some disagreement about which objectives are fundamental. Here, the union of all those objectives felt to be fundamental by some group member should be used. In the subsequent value model, the 'weight' that is placed on an objective on the list can be zero if a member feels that the specific objective is either irreverent or not fundamental.

\section{Identifying alternatives}

It is clear that the value of an alternative chosen for a decision can be no better than the value of the best alternative in the set of alternatives considered. Hence, except for the effort to appraise alternatives, the collaborative group can be no worse off with more alternatives. The collaborative group should encourage all members to create as many alternatives as they can. The collection of all of these alternatives should be the set considered in the decision. Each member should accept this, as their alternatives should be appropriately valued in a quality analysis. Any uneasiness about this would likely really concern the descriptions of consequences or the evaluation of consequences.

Because of the time and effort to evaluate alternatives with an analysis, it may be useful for the collaborative group to collectively eliminate alternatives that they feel are definitely inferior to at least one other on the list. Any procedure that the group felt was appropriate for this winnowing procedure would be fine to use.

\section{Describing consequences}

In describing consequences of alternatives, there are two situations to consider. The first one is when the members of the collaborative group can agree on a common description to relate alternatives to consequences. The second is when the group members do not agree on all the relationships so there are differences of judgements to consider.

Often different members of a group have experience concerning different objectives. Then the members with expertise on a given objective describe the part of each alternative's possible consequences related to that objective. I have worked on several decisions involving the selection of a site for a large energy facility (Keeney, 1980) and on an analysis to select a repository site for radioactive waste from nuclear power plants (Merkhofer and Keeney, 1987). The collaborative group agreed upon the consequences to describe alternatives in these cases. Regarding the repository, the collaborative group directed transportation analysts to build a model of the consequences due to transporting waste from all the commercial power plants to the different repository alternatives. 
The results included estimates of the transportation costs in dollars, the public and worker facilities due to transportation accidents, and the potential fatalities to the public and workers due to the possible exposure to the radioactive waste during transportation. Another team of biologists visited all the alternative sites and provided estimates of biological consequences that would occur if the repository were built at the site. Preliminary designs and plans for the construction of a repository at each site were developed by designers and used to develop estimates of economic costs for construction. When there are differences of opinion about the consequences that might result from at least some of the alternatives, it is first useful to understand these differences. At one extreme, each member of the collaborative group may have a different description of the consequences on each of the objectives. If this were the case, one could describe the consequences for each group member $M_{k}, k=1, \ldots, K$, with a distinct probability distribution $p_{j k}(x)$, which describes the possible consequences of alternatives $A_{j}$ using member $M_{k}$ 's judgement.

More likely, when there are disagreements, there may be only two or three viewpoints, each potentially described by a probability distribution for each alternative, where each group member believes one of the distributions. Even in this situation, there may be agreement on consequences of some of the alternatives and/or there may be point estimates for some of the consequences (i.e., a degenerate probability distribution with a probability of 1.0 describing the consequence).

When there are disagreements, the first step is to see if they can be reconciled using a deliberative process. The bases for the judgements should be discussed, learning can take place, and differences in judgement may be reduced. Clemen and Winkler (1999) discuss useful guidelines to constructively manage this deliberative process to reach consensus when appropriate and/or clarify remaining differences of judgement. Winkler (1983) and Winkler and Clemen $(1992,2004)$ discuss different methods for combining probabilities or point estimates when differences remain.

\section{Quantifying values}

Situations pertaining to the quantification of values are analogous to those for descriptions of consequences. The main difference is that only one utility function is needed to evaluate consequences, whereas a probability distribution function is needed for each alternative to describe consequences.

If all members of the collaborative group agree on a utility function, then that function should be used. This may happen when different members have expertise concerning the values of different levels of achievement on different objectives. For siting energy facilities, collaborative group members may defer their judgement to those of the biologists in specifying the relative values for different biological impacts and the value tradeoffs between economic costs and biological impacts (e.g., what cost is equivalent in value to the destruction of 1000 acres of mature pine forest).

The more complicated case is when collaborative team members disagree on some values and hence their values must be represented by different utility functions. As with the probability distributions for consequences, there often is only disagreement on some values and the differences may not be significant. In the extreme case where each group member has different values, a utility function $u_{k}, k=1, \ldots, K$. can be assessed for each individual. There are several models discussed in Keeney and Kirkwood (1975) and Keeney and Raiffa (1976) for combining utility functions of group members into a group utility function. Important details follow on this topic in the next Section 5. 


\section{Evaluating alternatives}

After gathering all the information needed for a decision analysis, there are again two situations. In the simpler case, which might be referred to as consensus, the collaborative group agrees on the descriptions of consequence and on the values to use for evaluating those consequences. These agreements are modelled in the probability distribution functions $p_{j}(x)$ for alternatives $A_{j}, j=1, \ldots, J$ and the utility function $u(x)$. Then, the analysis can proceed using equation (1) and a range of sensitivity analysis should be conducted that vary different parameters in the $p_{j}(x)$ and $u(x)$.

There is no requirement that a collaborative group reach consensus on the probability distributions describing consequences of alternatives or on the utility function to evaluate consequences. When no such consensus is reached, there are more than one $p_{j}(x)$ for some alternatives (i.e., different individuals have different viewpoints) and/or more than one $u(x)$, meaning different individuals have different values. In this case we want to evaluate alternatives for each individual using

$$
U_{j k}=\int p_{j k}(x) u_{k}(x) \mathrm{d} x,
$$

where $U_{j k}$ is the expected utility of alternative $A_{j}, j=1, \ldots, J$, to individual $M_{k}$, $k=1, \ldots, K$. In equation (2), if all individuals have common values, then $u$ is used for $u_{i}$ for each individual.

Given the set of $U_{j k}$ for all $j$ and $k$, one can extend the decision analysis to focus on how to best satisfy members of the collaborative group. For an individual $M_{k}$, the $U_{j k}$, $j=1, \ldots, J$ provides a rating, and hence implies also a ranking, of all the alternatives. One can examine these results for dominated alternatives. If one alternative is ranked lower than at least one other alternative by each individual, the lower ranked alternative is dominated and can be eliminated from further consideration. One might expect to find dominated alternatives because of the natural correlations that often occur among descriptions of consequences for individuals and among values of different individuals. This general process may result in identifying the real contenders for the best alternative or even sometimes clearly identify the best choice.

Sometimes, either the descriptions of consequences or the values may be different enough that a group 'aggregated analysis' is useful. For this, techniques to mathematically combine the different probability distributions, such as those in Clemen and Winkler (1999), and techniques to combine different group member's utility functions, such as those in Keeney and Raiffa (1976) could be used. The resulting aggregated functions should be used to evaluate alternatives using a wide array of sensitivity analysis to clearly understand the pros and cons of each alternative. Such analysis should provide insights to facilitate informed discussions that may select an alternative or at least make apparent some less desirable alternatives that can be dropped from further consideration.

\section{Aggregating preferences of individuals in collaborative groups}

This section focuses on one important aspect of implementing decision analysis for collaborative group decisions, namely the aggregation of preferences of the individual members within the collaborative group to derive a useful and usable group preference. 
The reason for this focus is a common misperception that such an aggregation is theoretically impossible.

A succinct characterisation of this aggregation problem is as follows. We want to obtain group preferences $P_{G}$ based on individual preferences $P_{k}, k=1, \ldots, K$. Specifically, we want to determine a function $f$ such that

$$
P_{G}=f\left(P_{1}, P_{2}, \ldots, P_{K}\right) .
$$

To determine $f$, a set of logical assumptions for combining individual preferences are stated, and then the hope is to derive $f$ such that it is consistent with these assumptions.

Arrow (1951) proved a famous result demonstrating that there is no $f$ satisfying one set of reasonable aggregation assumptions. This result, known as Arrow's Impossibility Theorem, has been misinterpreted by many (e.g., Hazelrigg, 1999; Franssen, 2005). They make a general conclusion that there is no reasonable or logical way to combine individual preference to obtain a group preference. But Arrow's result is more specific: it is for a specific type of preference, namely rankings, and a specific set of assumptions, namely the assumptions that he chose. Luce and Raiffa (1957) examine Arrow's result from many viewpoints.

This section presents results that logically combine individual preferences for the situation relevant to collaborative group decisions in this paper, namely the case where the preferences are von Neumann-Morgenstern utility functions. We begin with a summary of Arrow's formulation and result and then give the corresponding positive results for collaborative group decisions.

Arrow formulated his aggregation of preferences with $P_{G}$ and $P_{k}, k=1, \ldots, K$, being rankings of the set of alternatives $A_{j}, j=1, \ldots, J$. The logical assumptions that he wanted his aggregation to conform with are presented in Table 1 . Arrow then derived his impossibility theorem proving that there is no function $f$ in equation (3) that combines individual rankings for all possible sets of individual rankings consistent with his five assumptions.

This result does not directly apply to the case where preferences $P_{G}$ and $P_{k}$, $k=1, \ldots, K$ are von Neumann-Morgenstern expected utilities $U_{j}$ and $U_{j k}, k=1, \ldots, K$ respectively for alternatives $A_{j}, j=1, \ldots, J$. This is the case relevant to collaborative group decisions as framed in this paper because of the uncertainties necessary to describe the possible consequences of alternatives. Keeney (1976) kept the spirit of Arrow's assumptions, but altered them to pertain to von Neumann-Morgenstern expected utilities for alternatives. The set of analogous assumptions are presented in Table 2, where the group expected utility $U_{j}$ of an alternative is calculated using the individual's expected utilities $U_{j k}$. Using these assumptions, Keeney proved that

$$
U_{j}=\sum_{k=1}^{K} w_{k} U_{j k},
$$

where $w_{k}$ are positive weights for the individual's expected utilities. Technically, only two of the $w_{k}$ weights need be positive to avoid a contradiction with either the individual sovereignty or non-dictatorship assumptions. The expected utilities $U_{j}$ calculated using equation (4) provide a group rating of the alternatives consistent with the decision analysis assumptions and the assumptions for combining individual's preferences in Table 2. 
Table 1 Arrow's assumptions

A1 There are at least two individuals in the group, at least three alternatives, and a group
ranking is specified for all possible individual rankings
A2 (Positive association of social and individual values): If the group ranking indicates
alternative $a$ is preferred to alternative $b$ for a certain set of individual rankings, then the
group ranking must imply $a$ is preferred to $b$ if:
- the individual's ranking of alternatives other than $a$ are not changed
- each individual's ranking between $a$ and any other alternative either remains
unchanged or is modified in favour of $a$
(Independence of irrelevant alternatives): If an alternative is eliminated from
consideration, the new group ranking for the remaining alternatives should be equivalent
to the original group ranking for these same alternatives
A4 Individual's sovereignty): For each pair of alternatives $a$ and $b$, there is some set of
individual rankings such that the group prefers $a$ to $b$
A5 (Non-dictatorship): There is no individual such that whenever he prefers alternative $a$ to $b$,
the group will also prefer $a$ to $b$ regardless of the other individual's rankings

Arrow's Result: There is no way to combine individual rankings consistent with these assumptions.

Table 2 Keeney's assumptions

K1 There are at least two individuals in the group, at least two alternatives, and a group utility is specified for all possible individual utilities

K2 (Positive association of social and individual values): If the group utilities indicates alternative $a$ is preferred to alternative $b$ for a certain set of individual utilities, then the group utilities must imply $a$ is preferred to $b$ if:

- the individual's utilities of alternatives other than $a$ are not changed

- $\quad$ each individual's utilities between $a$ and any other alternative either remains unchanged or is modified in favour of $a$

K3 (Independence of irrelevant alternatives): If an alternative is eliminated from consideration, the new group utilities for the remaining alternatives should be equivalent to the original group utilities for these same alternatives

K4 (Individual's sovereignty): For each pair of alternatives $a$ and $b$, there is some set of individual utilities such that the group prefers $a$ to $b$

K5 (Non-dictatorship): There is no individual such that whenever he prefers alternative $a$ to $b$, the group will also prefer $a$ to $b$ regardless of the other individual's utilities

Keeney's Result: $U_{j}=\sum_{k=1} w_{k} U_{j k}$.

It is important to note that much earlier Harsanyi (1955) investigated formulation (3) where $P_{G}$ was a group utility function and the $P_{k}, k=1, \ldots, K$ were individual utility functions. He derived the additive group utility function $u_{G}$ from an appealing set of only three assumptions shown in Table 3, where two assumptions just refer to the existence of $u_{G}$ and the $u_{k}$ and the third assumption is substantive. This result can provide the group utility function $u$ needed in equation (1) to evaluate alternatives for a collaborative group. 
Table 3 Harsanyi's assumptions

\begin{tabular}{ll}
\hline H1 & Each $P_{k}, k=1, \ldots, K$, is a utility function $u_{k}$ over consequences $x$ \\
H2 & $P_{G}$ is a utility function $u_{G}$ over consequences $x$ \\
H3 & $\begin{array}{l}\text { If two alternatives, defined by probability distributions over the consequences } x, \\
\text { are indifferent to each individual, then they must be indifferent to the group }\end{array}$ \\
\hline
\end{tabular}

Harsanyi's Result: $u_{G}(x)=\sum_{k=1}^{k} w_{k} u_{k}(x)$.

The implications of these results are clear. One can derive group expected utilities for collaborative group decisions by aggregating the expected utilities of individual group members. This aggregation requires both the strength of preference information embedded in the utilities and interpersonal comparison of preferences addressed by the $w_{k}, k=1, \ldots, K$. Arrow did not want his formulation to include either. Subsequently, Sen (1970) demonstrated that formulations with the structure of equation (3) require interpersonal comparison of preferences in order to achieve a group preference for all possible sets of individual preferences.

\section{Uses of decision analysis for collaborative groups}

There are many situations where a collaborative group has responsibility for making a decision. Some of these decisions are very important. It is obviously worth making such decisions well as the only way to have a purposeful influence is by making decisions and carrying them out.

Important collaborative decisions are usually complex, as they typically involve multiple objectives, complicated technical or social interrelationships between alternatives and their consequences, and major uncertainties. There is no fundamental reason why all members of a collaborative group should agree on the importance of different objectives, on the relationships to describe consequences, or on these uncertainties. In other words, no consensus is necessary, and yet a decision must eventually be made.

Decision analysis can be of substantial help for such complex decisions faced by collaborative groups. It can help in several ways to

- $\quad$ organise the decision making process

- facilitate communication among group members

- evaluate alternatives

- provide insight for the decision.

The basic assumptions of decision analysis focus on the various elements necessary to make an informed decision. They organise the decision making process to separately address the objectives, alternatives, consequences, and values. The consistency assumptions indicate how to combine these elements to provide insight about the desireability of the various alternatives and their relative strengths and weaknesses.

The collaborative decision analysis process allows each group member to incorporate his or her knowledge, information, and judgement into the model. This makes explicit 
any differences about knowledge, information, and judgements held by the group members. Once clarified, many of these differences may be eliminated in productive discussions. For differences that remain, some may be eliminated by gathering some data or consulting outside experts that will lead to reconciliation of views. Even after this some differences may remain.

The evaluation of alternatives should be done separately for each of the members of the collaborative group. If there is consensus, the same evaluation is appropriate for each member. If there is no consensus, the different evaluations may provide some very useful insights. It is often the case that some alternatives can be eliminated from further consideration, as they are inferior to at least one other alternative using each of the group member's evaluations. This process should lead to focusing on the better alternatives or even may end up identifying the best alternative.

No model, or results of an analysis, ever makes a decision. The decision makers, meaning the collective group in our situation, must make the decision. The analysis informs them about what alternatives might be best and why. Sensitivity analysis can help significantly in this regard. Such sensitivity analysis, especially those examining alternatives using viewpoints of the different group members, provides insights for a constructive discussion about what decision the group should make.

For decisions where there is substantial disagreement about either or both the descriptions of the alternatives and the values to evaluate consequences. The decision analysis approach for collaborative group decisions transforms the focus from "which alternative should be chosen" to "how should an alternative be chosen given individual group members evaluations". The formulation discussed in Section 4 does this in a logical manner that explicitly incorporates and weighs the different viewpoints.

In summary, decision analysis provides a sound foundation and a helpful tool to guide collaborative group decisions. It does not oversimplify the realities of collaborative group decisions. It explicitly acknowledges differences of judgements and values among group members and offers a constructive framework to account for these differences in the decision process. Many collaborative group decisions need to be justified or explained to others including possibly bosses, customers, or the public. The record of a collaborative group decision analysis has everything needed to clarify the basis for a decision.

\section{References}

Arrow, K.J. (1951) Social Choice and Individual Values, 2nd ed., Wiley, New York, 1963.

Clemen, R.T. and Reilly, T. (2001) Making Hard Decisions with Decision Tools, Duxbury Press, Pacific Grove, California.

Clemen, R.T. and Winkler, R.L. (1999) 'Combining probability distributions from experts in risk analysis', Risk Analysis, Vol. 19, pp.187-203.

Franssen, M. (2005) 'Arrow's theorem, multi-criteria decision problems and multi-attribute preference in engineering design', Research in Engineering Design, Vol. 16, pp.42-56.

Harsanyi, J.C. (1955) 'Cardinal welfare, individualistic ethics, and interpersonal comparisons of utility', Journal of Political Economy, Vol. 63, pp.309-321.

Hazelrigg, G.A. (1999) 'An axiomatic framework for engineering design', Journal of Mechanical Design, Vol. 121, pp.342-347.

Keeney, R.L. (1976) 'A group preference axiomatization with cardinal utility', Management Science, Vol. 23, pp.140-145. 
Keeney, R.L. (1980) Siting Energy Facilities, Academic Press, New York.

Keeney, R.L. (1992) Value-Focused Thinking, Harvard University Press, Cambridge, Massachusetts.

Keeney, R.L. and Kirkwood, C.W. (1975) 'Group decision making using cardinal social welfare functions', Management Science, Vol. 22, pp.430-437.

Keeney, R.L. and Raiffa, H. (1976) Decisions with Multiple Objectives, Wiley, New York, Reprinted in 1993, Cambridge University Press, New York.

Luce, R.D. and Raiffa, H. (1957) Games and Decisions, Wiley, New York.

Merkhofer, M.W. and Keeney, R.L. (1987) 'A multiattribute utility analysis of alternative sites for the disposal of nuclear waste', Risk Analysis, Vol. 7, pp.173-194.

Pratt, J.W., Raiffa, H. and Schlaifer, R.O. (1964) 'The foundations of decision under uncertainty: an elementary exposition', Journal of the American Statistical Assosication, Vol. 59, pp.353-375.

Savage, L.J. (1954) The Foundations of Statistics, Wiley, New York.

Sen, A. (1970) Collective Choice and Social Warfare, Holden Day, San Francisco.

Tversky, A. (1969) 'Intransitivity of preferences', Psychological Review, Vol. 76, pp.31-48.

von Neumann, J. and Morgenstern, O. (1947) Theory of Games and Economic Behavior, 2nd ed., Princeton University Press, Princeton, New Jersey.

Winkler, R.L. (1983) 'The effects of combining forecasts and the improvement of the overall forecasting process', Journal of Forecasting, Vol. 2, pp.293, 294.

Winkler, R.L. and Clemen, R.T. (1992) 'Sensitivity of weights in combining forecasts', Operations Research, Vol. 40, pp.609-614.

Winkler, R.L. and Clemen, R.T. (2004) 'Multiple experts vs. multiple methods: combining correlation assessments', Decision Analysis, Vol. 1, pp.167-176. 